\title{
ESTIMATING UNCERTAIN REGIONS ON SMALL MULTIDIMENSIONAL DATASETS USING GENERALIZED PDF SHAPES AND POLYNOMIAL CHAOS EXPANSION
}

\author{
Maurice Imholz ${ }^{1}$, Dirk Vandepitte ${ }^{1}$, and David Moens ${ }^{1}$ \\ ${ }^{1}$ KU Leuven, Department of Mechanical Engineering \\ Celestijnenlaan 300B, 3001 Heverlee, Belgium \\ e-mail: maurice.imholz@kuleuven.be
}

Keywords: interval analysis, uncertainty quantification, polynomial chaos expansion, Bayesian inference

\begin{abstract}
In uncertainty analysis, estimating the degree of uncertainty based on some physical experiments is an essential part of the process to create robust products. Both at the input and the output side of an available model, experiments may be done, which can then be (inverserely) propagated to obtain uncertain results on the other side. In probabilistic analysis, PDF shape, stochastic moments and correlation may be inferred from this data. In possibilistic analysis, these quantities are hard to interpret physically and are therefore difficult to compute. Instead, interval bounds and dependency information can be determined. This paper presents a strategy to infer both interval bounds and dependency information from a (limited) set of data points in a multidimensional space, based on Polynomial Chaos Expansion and a generalized Probability Density Distribution (PDF) shape.
\end{abstract}




\section{INTRODUCTION}

The use of intervals in numerical modelling to represent uncertainty is gaining increased interest. Intervals can be more easily applied in cases of low data availability and where information on extreme cases is of higher inportance. The use of intervals to represent non-deterministic quantities omits the need to predefine a PDF, which may be hard to estimate when little data is available. However, the simplicity of intervals also has a large disadvantage: they are unable te represent dependency between different uncertain quantities. Some solutions to this have been the subject of recent research, such as interval fields [1, 2, 3, 5, 4], interactive fuzzy numbers [6, 7], interval correlation [8], and the use of copulas in interval context [9]. In this paper, a procedure is discussed that, given a small set of multidimensional data points, allows to make an estimate of the total population uncertainty, incorporating the dependency present in the data as well. It makes use of a generalized PDF shape to estimate intervals in a Bayesian inference scheme, and then Polynomial Chaos Expansion (PCE) to convert the hypercubic region spanned by the marginal interval estimates into a uncertain region of arbitrary shape that also incorporates the dependency present. Section 2 elaborates the concept of generalized PDF, also mentioned in [10], section 3 discusses the way the dependency is captured using PCE, and section 4 shows the combined method on a small 2D dataset.

\section{Bayesian Interval Estimation based on a generalized PDF shape}

This section provides a short summary of the method of estimating interval bounds based on a generalized PDF shape. The reader is refered to [10] for a more elaborate explanation.

\subsection{Principle of the generalized PDF shape}

Equation 1 shows the general formula for Bayesian analysis, which describes the posterior distribution given the data $\mathrm{D} p(\theta \mid D)$ as the product of the likelihood of the data and a prior distribution.

$$
p(\theta \mid D)=\frac{p(D \mid \theta) p(\theta)}{p(D)}
$$

$\theta$ represents a set of stochastic parameters that capture the PDF. In interval context, these parameters become the interval bounds $\bar{x}$ and $\underline{x}$. The data provides a minimum and maximum observed value $\widehat{x_{m}}$ and $\widehat{x_{M}}$. Equation 2 again describes Bayes' theorem, now in terms of the observed interval bounds and the true interval bounds that are to be estimated.

$$
p\left(\bar{x}, \underline{x} \mid \widehat{x_{M}}, \widehat{x_{m}}\right)=\frac{p\left(\widehat{x_{M}}, \widehat{x_{m}} \mid \bar{x}, \underline{x}\right) p(\bar{x}, \underline{x})}{p\left(\widehat{x_{M}}, \widehat{x_{m}}\right)}
$$

To express the likelihood function $p\left(\widehat{x_{M}}, \widehat{x_{m}} \mid \bar{x}, \underline{x}\right)$, an arbitrary PDF shape $S$ and corresponding stochastic parameter $\theta$ is introduced, as the interval bounds are assumed to bound an actual PDF shape, which cannot be identified properly because the dataset is too small. Equation 2 is then rewritten in terms of $S$ and $\theta$ as:

$$
\begin{aligned}
p\left(\widehat{x_{M}}, \widehat{x_{m}} \mid \bar{x}, \underline{x}\right) & =\int_{S} \int_{\theta} p\left(\widehat{x_{M}}, \widehat{x_{m}} \mid \theta\right) \cdot p(\theta \mid \bar{x}, \underline{x}) d \theta d S \\
& =\int_{S} \int_{\theta} M_{x}^{(n)}\left(\widehat{x_{M}}, \widehat{x_{m}}, \theta\right) \cdot p(\theta \mid \bar{x}, \underline{x}) d \theta d S
\end{aligned}
$$

this equation theoretically only holds if the integration is done over all possible PDF shapes $S$ and all values of the corresponding parameter value $\theta$. The first part of the integrand describes the occurence of certain extreme values given the PDF on the quantity $x$. This equals 
the extreme value distribution (EVD) $M_{x}^{(n)}\left(\widehat{x_{M}}, \widehat{x_{m}}, \theta\right)$, which depends on the number of experiments $n$. The second part describes the probability on having a certain stochastic parameter value, given the extreme bounds in the total population. Assuming the total population is very large, these extremes are equal to the maximum and minimum values allowed by the PDF that corresponds to a certain value of $\theta$. Given a certain bounded PDF shape $S$ (e.g. the uniform distribution, the 3- $\sigma$ bounded Gaussian distribution, ...), $p(\theta \mid \bar{x}, \underline{x})$ equals a delta function at that specific parameter value (or combination of values if more than one stochastic parameter is concerned) that puts the maximum and minimum possible values of the PDF at $\bar{x}$ and $x$. therefore, the integral needs to be taken over all possible PDF shapes $S$ that are bounded by $\bar{x}$ and $\underline{x}$ (equation 6).

$$
\begin{aligned}
p\left(\widehat{x_{M}}, \widehat{x_{m}} \mid \bar{x}, \underline{x}\right) & =\int_{S} \int_{\theta} M_{x}^{(n)}\left(\widehat{x_{M}}, \widehat{x_{m}}, \theta\right) \cdot \delta\left(\theta-\theta^{*}\right) d \theta d S \\
& =\int_{S} M_{x}^{(n)}\left(\widehat{x_{M}}, \widehat{x_{m}}, \theta^{*}\right) d S
\end{aligned}
$$

Putting this into equation 2, the interval Bayesian inference equation becomes:

$$
p\left(x^{I} \mid \widehat{x_{M}}, \widehat{x_{m}}\right)=\frac{\int_{S} M_{x}^{(n)}\left(\widehat{x_{M}}, \widehat{x_{m}}, \theta^{*}\right) d S \cdot p\left(x^{I}\right)}{p\left(\widehat{x_{M}}, \widehat{x_{m}}\right)}
$$

To be able to evaluate the integral, a generalized PDF shape is proposed based on 4 controleable parameters. By definition, $\int_{-\infty}^{+\infty} f_{x}(x) d x=\int_{x}^{\bar{x}} f_{x}(x) d x=1$. Many possible parametrizations are possible, and greatly influence the shapes that are considered in evaluating the integral. Since extreme values are of increased interest in the context of this paper, the following four control parameters are proposed (table 1), all focussing on the PDF shape in the extreme values.

\begin{tabular}{c|c} 
symbol & description \\
\hline$p_{0}$ & Probability density at $\underline{x}$ \\
$p_{1}$ & Probability density at $\bar{x}$ \\
$\left.\frac{d f_{x}(x)}{d x}\right|_{0}$ & first derivative of the PDF at $\underline{x}$ \\
$\left.\frac{d f_{x}(x)}{d x}\right|_{1}$ & first derivative of the PDF at $\bar{x}$
\end{tabular}

Table 1: parameters used to determine the PDF shape

A fourth order polynomial is proposed for the explicit representation of the PDF, given by equation 8 :

$$
f_{x}(x)=a x^{4}+b x^{3}+c x^{2}+d x+e
$$

Using this parameter set and the corresponding fourth order polynomial allows for a large variety of PDF shapes (including nonsymmetrical, sharp tailed, blunt tailed and bipolar shapes), while keeping the integral sufficiently fast to calculate.

The next section discusses two different ways of dealing with the integration defined in equation 7. Next to simply calculating it explicitly, it can also be bounded on the upside by calculating the maximum likelihood. 


\subsection{Average likelihood and worst-case likelihood estimation}

Returning back to equation 7, the following trivial relation can be established for the entire parameter set $\mathrm{S}$ (which has been defined above):

$$
M_{x}^{(n)}\left(\widehat{x_{M}}, \widehat{x_{m}}, \theta^{*}\right) \leq \max _{S} M_{x}^{(n)}\left(\widehat{x_{M}}, \widehat{x_{m}}, \theta^{*}\right)
$$

Integrating both sides gives:

$$
\int_{S} M_{x}^{(n)}\left(\widehat{x_{M}}, \widehat{x_{m}}, \theta^{*}\right) d S \leq \max _{S} M_{x}^{(n)}\left(\widehat{x_{M}}, \widehat{x_{m}}, \theta^{*}\right) \cdot \prod_{i=1}^{4}\left(\theta_{i, \max }-\theta_{i, \min }\right)
$$

This means that a conservative approximation of the integral can be calculated by determining the maximum likelihood value that occurs within the domain spanned by the parameters in table 1. Essentially, this means that for each test interval $x^{I}$, the likelihood is determined by the PDF shape that makes the observed interval most likely, which could be interpreted as the 'worst-case' PDF. Through this, more probability and therefore higher relative importance is given to larger intervals compared to explicitly evaluating the integral, which should lead to larger estimated intervals and therefore more conservative results. This paper refers to the latter approach as the worst-case likelihood (WCL) estimate, and the former as the average likelihood (AL) estimate. Previous testing of the method shows that the AL estimates tend to be not conservative enough to provide reliable results, but the WCL estimate, giving higher relative importance to larger intervals, does. The combined method discussed in section 4 will therefore use the interval Bayesian inference scheme illustrated here, using the WCL estimate to represent the likelihood function.

\section{Interval Polynomial Chaos Expansion}

In probabilistic analysis, Polynomial Chaos Expansion (PCE) [11] is used frequently to determine probability distributions on model output quantities. Application of PCE can be done in two ways:

- Given an output $y=f(x)$ as function of a random variable $x$ with known probability density function (PDF), the output distribution can be found by projecting onto a set of polynomial basis functions which are orthogonal w.r.t. the input PDF. Determining the output distribution then comes down to identifying the corresponding PC coefficients.

- Given a quantity $u$ with a known but complex PDF, its probability function can be described more easily by defining $u$ as a function of some germ variable $\xi$ with a simple PDF, such as the uniform or Gaussian distribution. Theoretically, by choosing the right functional relation, every type of distribution on $u$ can be obtained.

The first application is well established in the field of numerical modeling [12, 13]. The second application is of particular interest in sampling algorithms, as numerically sampling a complex PDF is nontrivial. Computers can effectively sample from the uniform or Gaussian distribution, but not necessarily from any arbitrary PDF.

\subsection{The Inverse Cumulative Density Function transform}

Using PCE, the objective is to obtain an explicit expression of:

$$
u=f(\xi)
$$


such that $u$ has a specific distribution, given the distribution of the germ variable $\xi$, which is a nontrivial problem, as many definitions of $f$ can lead to the required pdf. The question then becomes to find the most efficient one. It can be proven however that a solution for the above problem is always present, known as the inverse Cumulative Distribution Function (CDF) transform. If the PDF of $u$ is known and $u$ is a continuous variable, its CDF $F_{u}(u)$ exists and can be determined through:

$$
F_{u}(u)=\int_{-\infty}^{u} f_{u}(t) d t
$$

The domain of $F_{u}(u)$ is obviously $[0,1]$ as $f_{u}$ is always positive and $\int_{-\infty}^{\infty} f_{u}(t) d t=1$. We can then obtain the desired distribution of $u$ starting from a uniformly distributed germ between $[0,1]$ by writing:

$$
u=F_{u}^{-1}(\xi)
$$

For an arbitrarily distributed germ with $\mathrm{CDF}$ equal to $F_{\xi}$ we can write:

$$
u=F_{u}^{-1}\left(F_{\xi}(\xi)\right)
$$

This expression is especially useful because it holds for any distribution on $u$ or the germ $\xi$. However, it usually does not give the most efficient mapping.

\subsection{Dependent intervals}

The use of intervals is of particular interest in the presence of low data availability, as it omits the need of defining and quantifying a suitable PDF. An interval $x^{I}=\langle\underline{x} \mid \bar{x}\rangle$ only requires an upper and lower bound to be defined and describes a continuous region of possible values for the quantity $x$. On the probability of occurence within the interval, no assumption is made, and for the purpose of interval analysis, the probability is assumed to be nonzero of the interval interval, and strictly zero elsewhere. In the multivariate case, an interval vector $\mathbf{x}^{\mathbf{I}}=\left[x_{1}^{I} x_{2}^{I} \cdots x_{N}^{I}\right]$ is used with each entry a simple interval variable. By definition, the entries are assumed independent, so the interval vector defines a set of vectors in the $N$-dimensional space described by:

$$
\mathbf{x}^{\mathbf{I}}=\left[\hat{\mathbf{x}} \mid \hat{x}_{1} \in x_{1}^{I}, \hat{x}_{2} \in x_{2}^{I}, \cdots, \hat{x}_{N} \in x_{N}^{I}\right]
$$

Equation 15 describes a hypercube in the $N$-dimensional domain. Figure 1 illustrates this. If two interval parameters $a^{I}$ and $b^{I}$, are independent, this representation is accurate and introduces no further conservativity. However, in the other case, some degree of conservativity is always introduced through modelling with an interval vector. The higher the dependency, the more conservative this representation will be. This problem cannot be solved within the simple definition of an interval, as not enough parameters are available to represent dependency. Also, this dependency may take a large variety of forms, depending on the shape of the region of possible $(a, b)$-couples, so the uncertainty model would need a large amount of extra parameters to account for this. Still, the conservativity issue remains and should be addressed.

In structural dynamics, propagation of input interval parameters is usually done through optimization and anti-optimization within the region defined by the input intervals, as the models are usually quite complex and non-monotonous behaviour is possible. Since intervals make no assumption on the probability within the region the define, the optimization is supposed to be unbiased and treat all points in the region as equally probable. For this reason, intervals are usually sampled in a uniform way within the purpose of finding the output optima. So allthough 

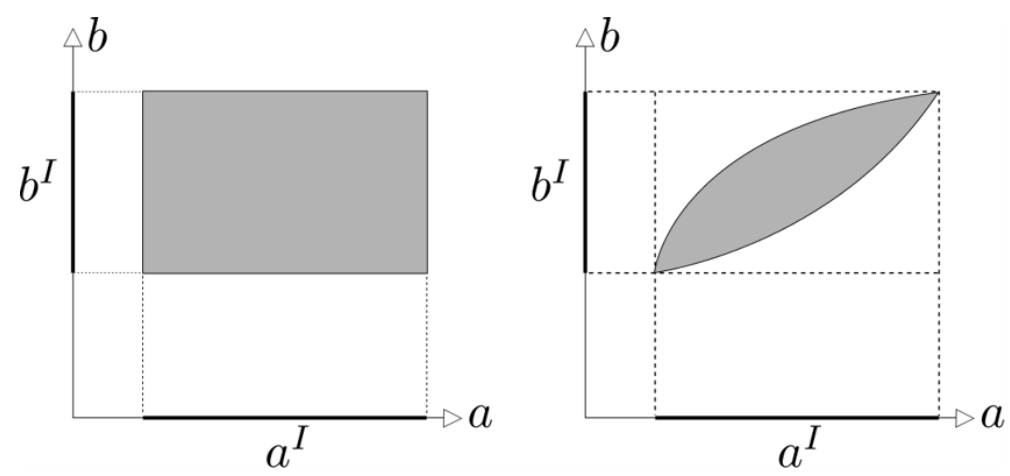

Figure 1: illustration of independent (left) versus dependent (right) interval quantities. The independent case is characterized by the hypercubic region, while the dependent case can theoretically consider any region.

the exact probability is unknown, the uniform distribution is assumed in practise for the purpose of propagating the uncertainty.

The same principle holds in the multivariate case. Given a region with dependency as shown in the right side of figure 1, the sampler is supposed to be unbiased towards any part of the region. This adds an extra requirement to the accurate definition of the uncertain region. Not only does the boundary of the region have to be represented accurately, but also within the region itself uniform sampling has to be possible. The next section describes a PCE-inspired technique that can capture a wide variety of dependencies, starting from a simple interval vector, and incorporating the possibility of uniform sampling, which is called interval PCE. The method will first be illustrated in $2 \mathrm{D}$, but can be theoretically expanded to any number of interval components.

\section{3 interval PCE}

Consider two interval parameters $x_{1}^{I}$ and $x_{2}^{I}$, with corresponding interval bounds $x_{1}, x_{2}$ and $\overline{x_{1}}, \overline{x_{2}}$. Assume some dependency is present, which is characterised by a region $\Omega$. Assume the bounds of the intervals itself are perfectly non-conservative, so the square described by the interval vector $\mathbf{x}^{\mathbf{I}}=\left[x_{1}^{I} x_{2}^{I}\right]$ is the smallest circumscribed square still fully encapsuling $\Omega$. The means that the far left and far right point part of the region are given by $\left(\underline{x_{1}}, x_{2, l e f t}\right),\left(\overline{x_{1}}, x_{2, \text { right }}\right)$. The upper and lower bounding curve of the region are distinguished as $C_{u}: x_{2}=H\left(x_{1}\right)$ and $C_{l}: x_{2}=h\left(x_{1}\right)$ in between these points (see also figure 2). The PCE is then one of the following form:

$$
\left\{\begin{array}{l}
x_{1}^{*}=F_{\Omega}^{-1}\left(\frac{x_{1}-\underline{x_{1}}}{\overline{x_{1}}-\underline{x_{1}}}\right) \\
x_{2}^{*}=\left(H\left(x_{1}\right)-h\left(x_{1}\right)\right) \overline{\frac{x_{2}}{x_{2}}-\underline{x_{2}}}+h\left(x_{1}\right)
\end{array}\right.
$$

with

$$
F_{\Omega}\left(x_{1}\right)=\frac{1}{A} \int_{\underline{x_{1}}}^{x_{1}} d t_{1} \int_{h\left(t_{1}\right)}^{H\left(t_{1}\right)} d t_{2}
$$

In equation 17, $A$ is the total surface area of $\Omega$, and the double integral describes the surface area of the part of $\Omega$ left of a certain value $x_{1}$. Essentially, the first line of equation 16 is an inverse CDF transform: $x_{1}^{*}$ is given an artificial distribution, which increases with increasing range of possible $x_{2}$-values at a certain value for $x_{1}$. This ensures that if $x_{1}$ and $x_{2}$ are sampled uniformly, the corresponding points are uniformly distributed over the domain $\Omega$. The second line describes a very simple transformation from a uniform distribution between $\overline{x_{2}}$ and $\underline{x_{2}}$ to a 


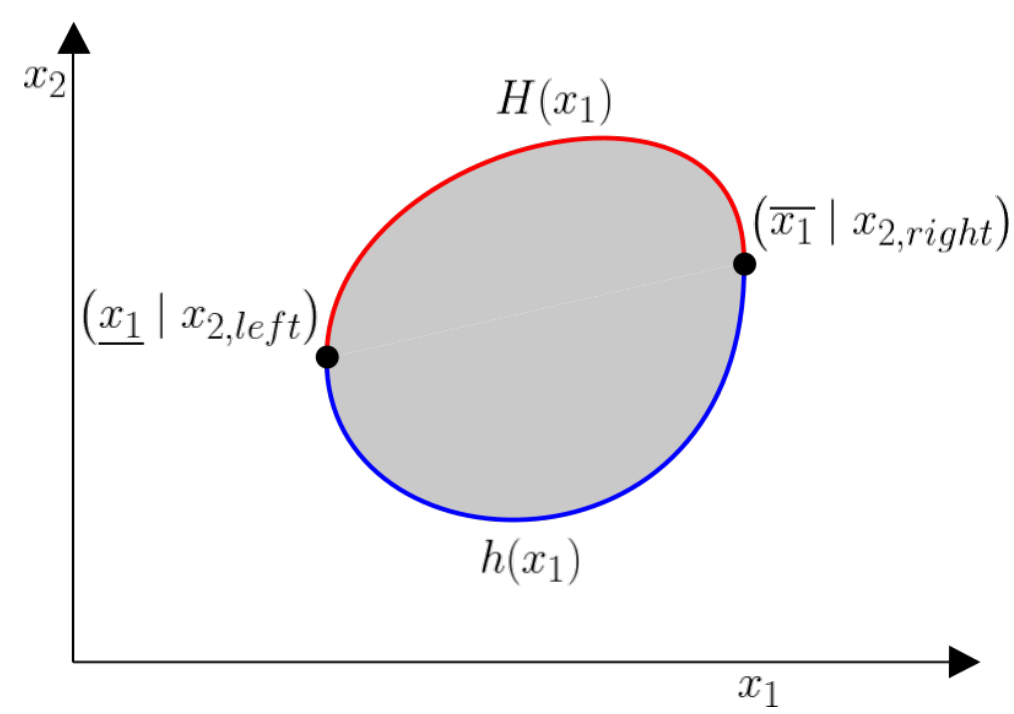

Figure 2: illustration of the quantities mentioned in the following equations

uniform distribution between the upper and lower bounding curve $C_{u}$ and $C_{l}$, for a certain value of $x_{1}$.

In multidimensional space, the PCE is of the following form:

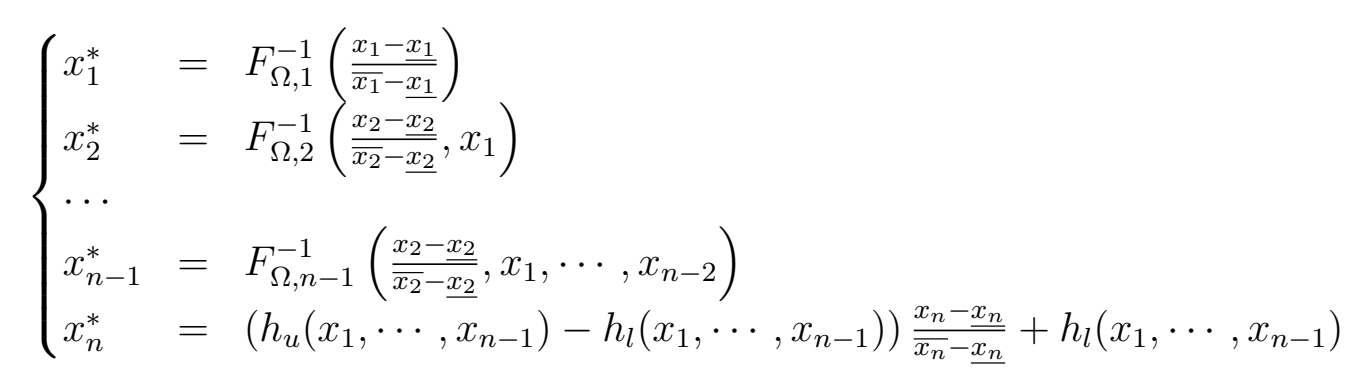

with

$$
\begin{cases}F_{\Omega, 1}\left(x_{1}\right) & =\frac{1}{\Omega_{n}} \int_{x_{1}}^{x_{1}} d t_{1} \int_{h_{1}\left(t_{1}\right)}^{H_{1}\left(t_{1}\right)} d t_{2} \int_{h_{2}\left(t_{1}, t_{2}\right)}^{H_{2}\left(t_{1}, t_{2}\right)} d t_{3} \cdots \int_{h_{n-1}\left(t_{1}, \cdots, t_{n-1}\right)}^{H_{n-1}\left(t_{1} \cdots, t_{n-1}\right)} d t_{2} \\ F_{\Omega, 2}\left(x_{2}, x_{1}\right) & =\frac{1}{\Omega_{n-1}} \int_{h_{1}\left(x_{1}\right)}^{x_{2}} d t_{2} \int_{h_{2}\left(x_{1}, t_{2}\right)}^{H_{2}\left(x_{1}, t_{2}\right)} d t_{3} \cdots \int_{h_{n-1}\left(x_{1}, t_{2}, \cdots, t_{n-1}\right)}^{H_{n-1}\left(x_{1}, t_{n} \cdots, t_{n-1}\right)} d t_{n} \\ \cdots & =\frac{1}{A} \int_{h_{n-2}\left(x_{1}, \cdots, x_{n-2}\right)}^{x_{n-1}} d t_{n-1} \int_{h_{n-1}\left(x_{1}, \cdots, x_{n-2}, t_{n-1}\right)}^{H_{n-1}\left(x_{1}, \cdots, x_{n-2}, t_{n-1}\right)} d t_{n} \\ F_{\Omega, n-1}\left(x_{n-1}, \cdots, x_{1}\right) & \end{cases}
$$

In equation 18, the inversion is only done with respect to the first variable inside the braccets, leading to stairwise dependency in the expanded quantities as $x_{1}^{*}=f\left(x_{1}\right), x_{2}^{*}=f\left(x_{1}, x_{2}\right), x_{3}^{*}=$ $f\left(x_{1}, x_{2}, x_{3}\right)$ and so on. This expansion requires an explicit formula for the edge of the region, which can be hard to construct in higher dimensional space, particularly finding explicit descriptions of $H_{i}$ and $h_{i}$ in equation 19 . Quite often, only 2-way interactions are considered in high dimensional spaces as they tend to have a higher relative impact on the output, which comes down to capturing the dependency in 2D-projections of the total uncertain space. Therefore, the 2D-case is considered in the remainder of this paper.

Usually, an explicit description for $F_{\Omega}\left(x_{1}\right), C_{u}$ and $C_{l}$ is not available, or very difficult to express, so in practise they are expressed by using a truncated PCE based on the univariate legendre polynomials, as they are orthogonal w.r.t. the uniform distribution. 


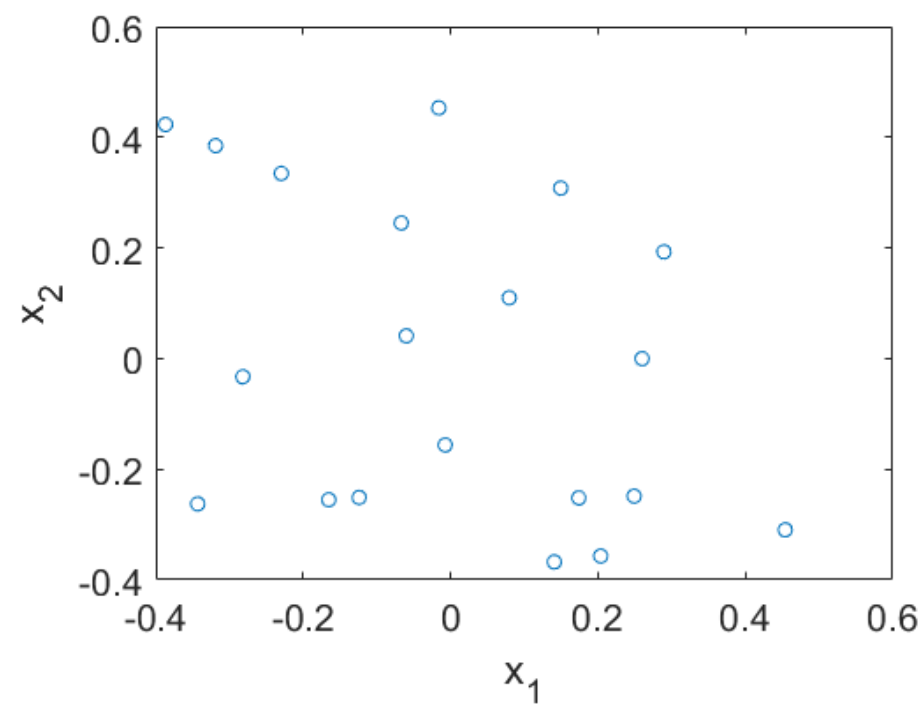

Figure 3: data points used in this example of the combined method

\section{The combined method to uncertain regions from small datasets}

The method in this section is explained by applying it on the virtual dataset of 20 points, as is given in figure 3 .

The data is captured in the $20 \times 2$ matrix $\mathbf{X}$. The objective will be to estimate an uncertain region on it in an interval context. The dataset is centered and the eigenvectors $\Phi$ of $\mathbf{X}^{\mathbf{T}} \mathbf{X}$ are identified. The data is projected onto both eigenvectors, leading to two projected sets $\mathbf{u}_{1}=\mathbf{X} \phi_{1}$ and $\mathbf{u}_{2}=\mathbf{X} \phi_{2}$. Next to this two additional projections are done on the vectors $\mathbf{v}=\frac{\lambda_{1} \phi_{1}+\lambda_{2} \phi_{2}}{\sqrt{\lambda_{1}^{2}+\lambda_{2}^{2}}}$ and $\mathbf{w}=\frac{\lambda_{1} \phi_{1}-\lambda_{2} \phi_{2}}{\sqrt{\lambda_{1}^{2}+\lambda_{2}^{2}}}$. Projection on these vectors lead to two additional sets $\mathbf{u}_{3}=\mathbf{X v}$ and $\mathbf{u}_{4}=\mathrm{Xw}$. These directions signify the two height lines and diagonals of the smallest circumferential rectangle of the dataset. On all four of the projected datasets, the bayesian inference scheme as was described in section 2 is performed, leading to a total of 4 estimated intervals, which are then multiplied with their corresponding direction, leading to 8 points in the $2 \mathrm{D}$-space that serve as the boundary points of the uncertain region. For the uncertain region, the following parametrization is used (equation 20):

$$
\left\{\begin{array}{l}
x_{1}=R(\theta) \cos (\theta) \\
x_{2}=R(\theta) \sin (\theta)
\end{array}\right.
$$

with

$$
\begin{aligned}
R(\theta) & =a_{0}+a_{1} \cos (\theta)+a_{2} \sin (\theta)+a_{3} \cos (2 \theta) \\
& +a_{4} \sin (2 \theta)+a_{5} \cos (3 \theta)+a_{6} \sin (3 \theta)+a_{7} \cos (4 \theta)
\end{aligned}
$$

The coefficients $a_{0}$ to $a_{7}$ can be uniquely determined by the 8 boundary points, leading to the uncertain region in figure 4

The second part of the method is to apply the theory of interval PCE as described in section 2 to ensure the mapping of the initial variables to the expanded parameters $x_{1}^{*}$ and $x_{2}^{*}$ ensure that not only the hypercubic spaces is transformed into the uncertain region that was just determined, but also that a uniform sampling on the initial variables also produce a uniform sampling of the uncertain region. The most left and right points are determined by solving $\frac{\partial x_{1}}{\partial \theta}=0$, which is 


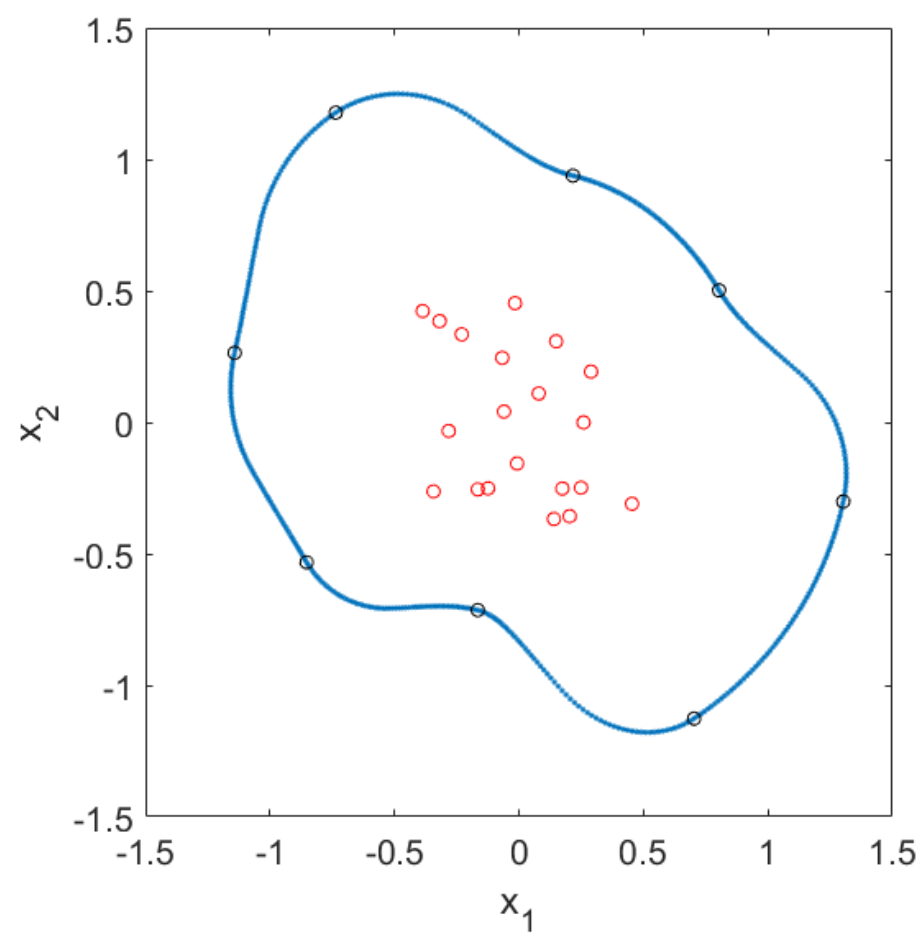

Figure 4: Curve fitted using the 8 boundary points (black circles) and the parametrization in equation 20 and 21

valid for exactly 2 values of $\theta$, referred to as $\theta_{l}$ and $\theta_{r}$. The upper and lower curve are discretized and fitted using a 10th order Legendre polynomial set, the result of which is given in figure 5 .

The CDF as described in equation [17] is determined and inverted numerically and is fitted using a 10th order Legendre polynomial set, the result of which is given in figure 6

This gives an expression for the mapping of $x_{1}$ and $x_{2}$ on $x_{1}^{*}$ and $x_{2}^{*}$ given by equation 22 . The coefficient values are given in table 2 .

$$
\left\{\begin{array}{l}
x_{1}^{*}=\sum_{i=0}^{10} c_{i} \cdot P_{i}\left(\frac{x_{1}-\underline{x_{1}}}{\overline{x_{1}}-\underline{x_{1}}}\right) \\
x_{2}^{*}=\left(\sum_{i=0}^{10}\left(H_{i}-h_{i}\right) \cdot P_{i}\left(x_{1}^{*}\right)\right) \frac{x_{2}-\underline{x_{2}}}{\overline{x_{2}}-\underline{x_{2}}}+\sum_{i=0}^{10} h_{i} \cdot P_{i}\left(x_{1}^{*}\right)
\end{array}\right.
$$

Table 2: coefficient values in equation 22 (values are multiplied by 100)

\begin{tabular}{l|lllllllllll}
\hline$i=$ & 0 & 1 & 2 & 3 & 4 & 5 & 6 & 7 & 8 & 9 & 10 \\
\hline$H_{i}$ & -67.06 & 18.67 & 53.65 & -22.16 & -7.47 & 3.98 & 13.0 & 7.10 & -0.58 & -10.82 & 2.06 \\
$h_{i}$ & 78.36 & 24.43 & -44.17 & -16.69 & -14.7 & -8.45 & -3.52 & 6.62 & -5.67 & -3.63 & -0.45 \\
$c_{i}$ & 50.66 & 42.69 & 0.14 & 4.45 & -0.70 & 1.07 & -0.21 & 0.64 & 0.23 & 0.53 & -0.08 \\
\hline
\end{tabular}

In equation 22, $P_{n}(x)$ refers to the $n$th order Legendre polynomial. The final result of the method given by equation 22 and figure 7 illustrates a uniform sampling within the bounds of the basic variables and the result in the transformed space of $x_{1}^{*}$ and $x_{2}^{*}$.

\section{Conclusion}

This work presented a method to estimate two-dimensional uncertain regions, accounting for possible dependency between the uncertain quantities. From figure 7, it can be seen that 


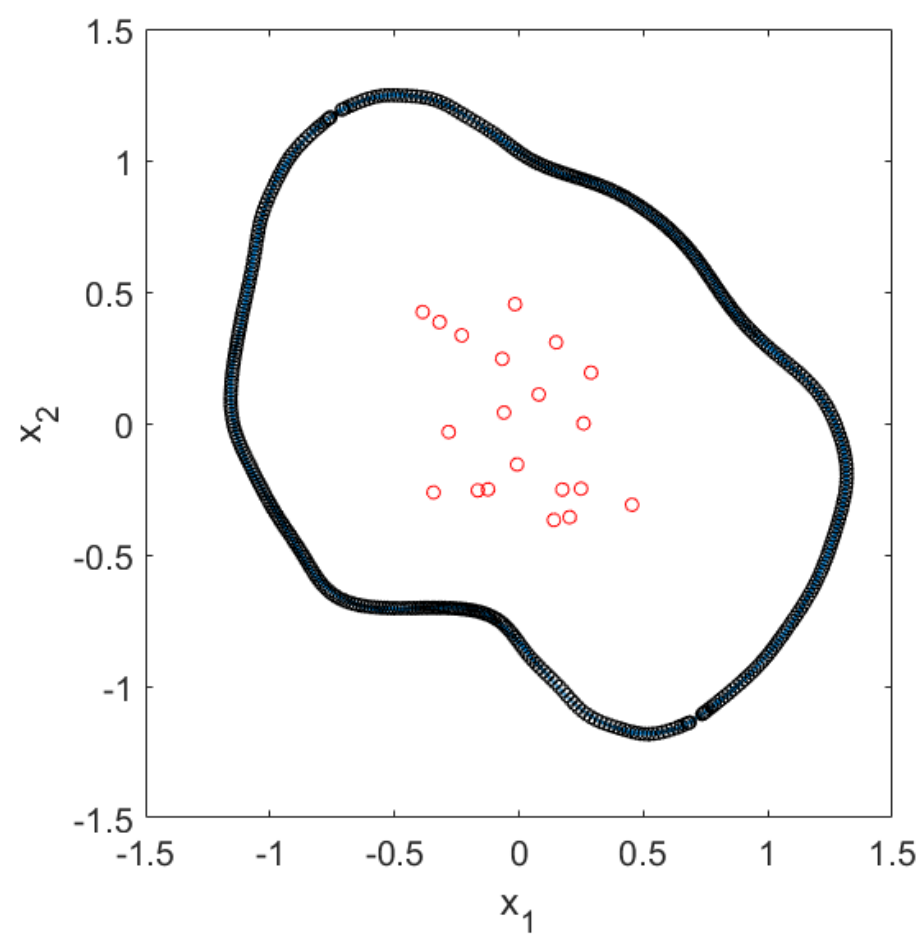

Figure 5: approximation of the upper and lower curve using a 10th order Legendre polynomial set. Blue dots: real curve, black circles: fitted curve

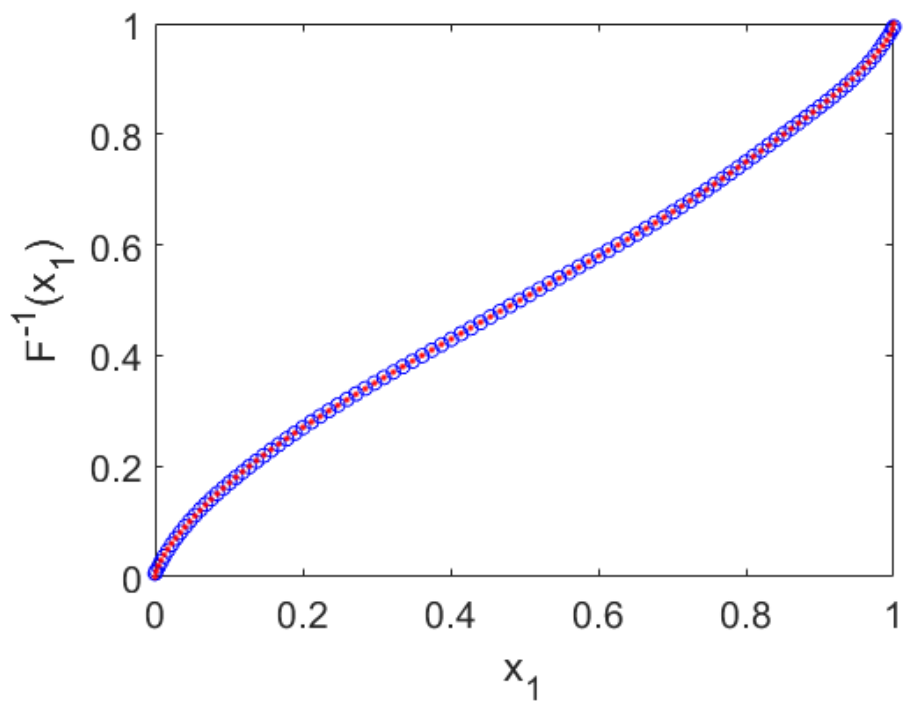

Figure 6: Inverted CDF, red dots: actual curve determined by integral evaluation, blue circles: fitted curve 

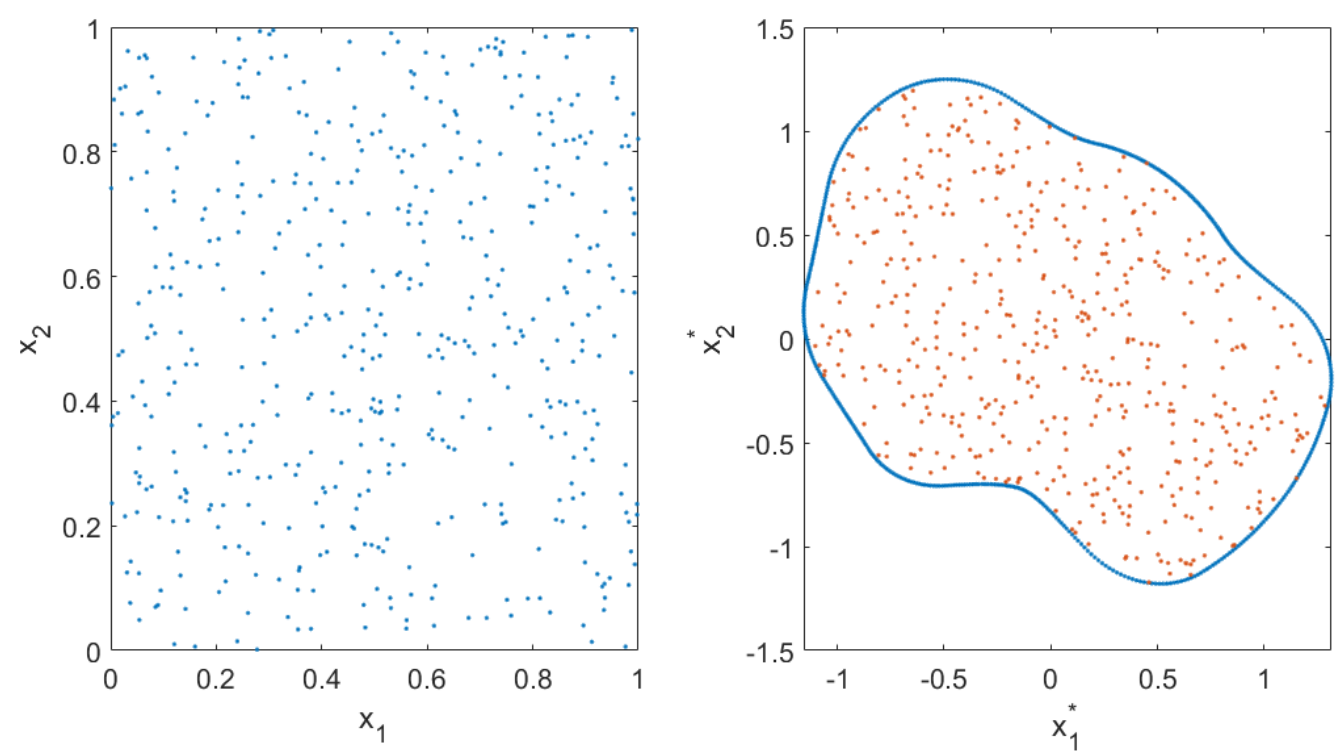

Figure 7: 500 points uniformly sampled from a non-interactive normalized uncertain space (left) and the resulting uncertain region (right)

from a uniform sampling of the initial germ variables, a uniform distribution of the transformed quantities is obtained, and the boundary curve is obeyed as well. Future work will be done on applying the combined method on actual datasets on Finite Element model input parameters and compute resulting output uncertainty, and perform (anti-)optimization to obtain bounds on uncertain output quantities.

\section{Acknowledgements}

The authors gratefully acknowledge financial support from FWO-Vlaanderen (the Flemish fund for scientific research) and from the KU Leuven research fund. This work is done with funds provided by FWO project No. G0C2218N, High Dimensional Interval Fields: spatial uncertainty modelling for mechanical reliability (HiDiF).

\section{REFERENCES}

[1] D. Moens, M. De Munck, W. Desmet, D. Vandepitte, Numerical dynamic analysis of uncertain mechanical structures based on interval fields, IUTAM Symposium on the Vibration Analysis of Structures with Uncertainties. IUTAM Bookseries, vol 27. (2011), pp. 71-83, Springer, Dordrecht

[2] W. Verhaeghe, W. Desmet, D. Vandepitte, D. Moens, Interval fields to represent uncertainty on the output side of a static FE analysis, Computer Methods in Applied Mechanics and Engineering, vol. 206 (2013), pp. 50-62

[3] M. Imholz, D. Vandepitte, D. Moens, Derivation of an input interval field decomposition based on expert knowledge using locally defined basis functions, Proceedings of the 1st International Conference on Uncertainty Quantification in Computational Sciences and Engineering, UNCECOMP2015, pp. 529-547, May 2015, Crete, Greece 
[4] A. Sofi, E. Romeo, O. Barrera, A. Cocks, An interval finite element method for the analysis of structures with spatially varying uncertainties, Advances in Engineering Software, vol. 128 (2019), pp. 1-19

[5] W. Gao, D. Wu, K. Gao, X. Chen, F. Tin-Loi, Structural reliability analysis with imprecise random and interval fields, Applied Mathematical Modelling, vol. 55 (2018), pp. 49-67

[6] R. Fuller, P. Majlender, On interactive fuzzy numbers,Fuzzy Sets and Systems, vol. 143 (2004), pp. 355-369

[7] J.J. Buckley, On the algebra of intervactive fuzzy numbers, Fuzzy Sets and Systems, vol. 32 (1989), pp. 291-306

[8] P. Pandian, K. Kavitha, On correlation between two real interval sets, Journal of Physics: conf. series 1000012055

[9] M. Faes, D. Moens, High dimensional dependence via pair constructions for interval finite models, USD2018 International conference on uncertainty in Structural Dynamics, September 2018, Leuven

[10] M. Imholz, D. Vandepitte, D. Moens, Bayesian estimation of interval bounds based on limited data, USD2018 International conference on uncertainty in Structural Dynamics, September 2018, Leuven

[11] A. O'Hagan, Polynomial Chaos: A Tutorial and Critique from a Statistician's Perspective, SIAM/ASA Journal on Uncertainty Quantification, 2013

[12] G. Blatman, B. Sudret, Adaptive sparse polynomial chaos expansion based on least squares regression, Journal of Cumputational Physics, vol. 230 (2011), pp. 2345-2367

[13] Z. Ma, J. Wu, Y. Zhang, M. Jiang, Recursive parameter estimation for load sensing proportional valve based on polynomial chaos expansion, Engineering Computations, Vol.32 (2015), pp.1343-1371 